\title{
A new astrometric measurement and reduction of USNO photographic observations of Phobos and Deimos: 1967-1997^
}

\author{
V. Robert ${ }^{1,2}$, V. Lainey ${ }^{2}$, D. Pascu ${ }^{3, \star \star}$, A. Pasewaldt ${ }^{4}$, J.-E. Arlot ${ }^{2}$, J.-P. De Cuyper ${ }^{5}$, V. Dehant ${ }^{5}$, and W. Thuillot ${ }^{2}$ \\ ${ }^{1}$ Institut Polytechnique des Sciences Avancées IPSA, 11-15 rue Maurice Grandcoing, 94200 Ivry-sur-Seine, France \\ 2 Institut de Mécanique Céleste et de Calcul des Éphémérides IMCCE, Paris observatory, UPMC, Univ. Lille 1, CNRS, \\ 77 Av. Denfert-Rochereau, 75014 Paris, France \\ ${ }^{3}$ United States Naval Observatory USNO, 3458 Massachusetts Ave NW, Washington, DC 20392, USA \\ 4 Technische Universität Berlin TUB, Department of Geodesy and Geoinformation Science, Strasse des 17. Juni 135, 10623 Berlin, \\ Germany \\ 5 Royal Observatory of Belgium ROB, avenue Circulaire 3, 1180 Uccle, Belgique
}

Received 16 July 2015 / Accepted 9 August 2015

\section{ABSTRACT}

\begin{abstract}
Context. Accurate positional measurements of planets and satellites are used to improve our knowledge of both their orbits and their dynamics and to infer the accuracy of the planet and satellite ephemerides.

Aims. In the framework of the European FP7 ESPaCE program, we provide the positions of Mars, Phobos, and Deimos taken with the US Naval Observatory 61-inch astrometric reflector and 26-inch refractor from 1967 to 1997.

Methods. Four hundred twenty five astrophotographic plates were measured with the digitizer of the Royal Observatory of Belgium and reduced through an optimal process that includes image, instrumental, and spherical corrections using the UCAC4 catalog to provide the most accurate equatorial (RA, Dec) positions.

Results. We compared the observed positions of the planet Mars and its satellites with the theoretical positions from INPOP13c and DE430 planetary ephemerides and from NOE MarsSatV1_0 and MAR097 satellite ephemerides. The rms residuals in RA and Dec of one position are less than 62 mas or about $20 \mathrm{~km}$ at the opposition of Mars. The rms intersatellite residuals in RA and Dec of one position are less than 40 mas or about $13 \mathrm{~km}$ at Mars. This accuracy is comparable to the most recent CCD observations. We also fitted the NOE model to the new computed positions and compared the orbital evolution of Phobos and Deimos with those derived from the same model, but only fitted to spacecraft data. Our results show that astrophotographic plate data can now compete with those of old spacecraft.
\end{abstract}

Key words. astrometry - ephemerides - planets and satellites: individual: Phobos - planets and satellites: individual: Deimos

\section{Introduction}

The European Satellites Partnership for Computing Ephemerides (ESPaCE) project aims at strengthening the collaboration and at developing new knowledge, new technology, and products for the scientific community in the domains of the development of ephemerides and reference systems for natural satellites and spacecraft. Several European research centers involved in space sciences and dynamics were associated. The activity is focused on extracting and analyzing astrometric data from spacecraft and ground-based measurements that will be combined to build new ephemerides. It is intended to provide new dynamical models for natural satellites and a characterization of their rotation properties, and to improve spacecraft orbit determination from space science. Since we had demonstrated that a precise digitization and a new astrometric reduction of old photographic plates could provide very accurate

* Full Table 2 and Tables of the $X Y$ positions of the satellites and their references are available in electronic form at the CDS via anonymous ftp to cdsarc.u-strasbg.fr (130.79.128.5) or via http://cdsarc.u-strasbg.fr/viz-bin/qcat?J/A+A/582/A36, and at the Natural Satellites Data Center service of IMCCE via http://www.imcce.fr/nsdc/

$\star \star$ USNO retired. positions (Robert 2011; Robert et al. 2011), the project leaders chose to consider such observations as a significant task for the ESPaCE program.

Recently, Desmars et al. (2009) have shown that astrometric data spread over a large time span were better than more accurate astrometric data spread over a short interval of time for dynamical and ephemeris purposes. The extrapolation of the ephemerides for space missions are more confident. Similarly, Arlot et al. (2012) show the benefit of using old observations and show how new reductions and future reductions with the Gaia catalog (Robin et al. 2012) will be useful.

In view of this, we have obtained the large photographic plate archive of the Martian satellites taken at the USNO from 1967 to 1997 for remeasurement and reanalysis. From a one-opposition sample of this plate archive, we demonstrated that we could provide accurate positions for Mars and its satellites (Robert et al. 2014) and decided to reanalyze the complete 30 -yr series.

\section{Historical context}

Following their discovery by Asaph Hall in 1877 (Hall 1878) with the USNO 26-inch refractor, the two Martian moons were observed regularly near the time of Mars' favorable oppositions, which occur every 15 or $17 \mathrm{yr}$. The observational technique of 
choice was the long-focus visual refractor, mounted with a filar micrometer. Such observations continued until the opposition of 1924 and thereafter declined.

By the early 1940s, the micrometer observations ceased altogether as interest in the satellites waned. However, the report by Sharpless (1945) of the detection of the long suspected secular accelerations in the longitudes of the satellites revived interest in them. When a plausible theory for the accelerations could not be found (Burns 1972), the veracity of the accelerations was questioned. Using all astrometric observations of the Martian satellites, Wilkins $(1966,1967,1970)$ attempted to confirm Sharpless' values for the accelerations but was unsuccessful. Wilkins suggested that the observations were not accurate enough to solve for the accelerations and made a plea for new observations.

Amid reports of large ephemeris errors and questionable accelerations, the USNO began a thirty-year (1967-1997) program of photographic observations of the Martian satellites (Pascu 1977, 1978, 1979, 2012). These observations were among the most accurate (Morley 1989) and were used to support all space reconnaissance projects of the Martian system, as well as dynamical studies of the satellite motions (Lainey et al. 2007). Two drawbacks to the program were that the photographic plates were manually measured because the satellites were embedded in the planetary halo, and the trail/scale method was used in the reductions (Pascu 1977) since accurate positions for the faint stars on the plates were not available at the time. With the construction of the high precision/high speed DAMIAN digitizing measuring machine (de Cuyper et al. 2011) and the construction of dense, high precision star catalogs such as UCAC4 (Zacharias et al. 2013), these drawbacks were no longer an issue, and it appeared promising to not only improve the accuracy of the satellite observations, but also to obtain accurate equatorial (RA, Dec) positions for the planet itself.

Our strategy was to use the methods developed in the analysis of the USNO Galilean plates (Robert 2011; Robert et al. 2011) to analyze a set of USNO Martian satellite observations, determining equatorial (RA, Dec) positions for Mars and its moons. The success of this initiative (Robert et al. 2014) motivated us to analyze the complete set of these observations.

\section{The USNO observations}

Photographic observations were begun in 1967 and continued at every opposition through 1997 with the exception of the opposition of 1993 because of poor weather. This includes all favorable oppositions and unfavorable oppositions. Oppositions occur, on average, every 26 months, and favorable oppositions occur every 15 or $17 \mathrm{yr}$. The scale of the Martian system at a favorable opposition is about twice that at the most unfavorable. Because the 1967 and 1969 oppositions were not favorable, the photographic plates were taken with the USNO 61-inch astrometric reflector in Flagstaff, Arizona. This instrument has a focal scale of $13.551 \mathrm{arcsec} / \mathrm{mm}$. Most of the plates, however, were taken with the 26-inch refractor in Washington, DC. This instrument has a focal scale of $20.851 \mathrm{arcsec} / \mathrm{mm}$ at full aperture.

The entire 31-yr series was characterized by the use of special filters. Several Schott $5 \times 7$ inch $\times 3$ mm GG14 (yellow) filters were polished optically flat. In the center of each a small, thin metallic nichrome film was deposited by evaporation (Fredrick 1966, priv. comm.) with an optical density of about 3.0. Either a round "spot" or lozenge shape was made to accommodate all oppositions, as well as both the
USNO 61-inch reflector in Flagstaff and USNO 26-inch refractor in Washington, DC. The GG14 was chosen to accommodate both telescopes, and nichrome was chosen because it transmits neutrally in the visual bandwidth. The function of the small nichrome filter was to reduce the intensity of the planetary image to that of the satellites, producing a measurable image of the planetary disk. This made it possible to obtain positions of each satellite, independently avoiding, when possible, some of the drawbacks of intersatellite observations, such as high correlations in the orbital correction solutions. It also made it possible to obtain observations of one satellite when the other one was not available or well defined. And finally, the equatorial position of the planet could be obtained even when images of the satellites were unavailable (Pascu 1977). A cartridge plateholder was built in which the GG14 filter was fixed in $2 \mathrm{~mm}$ above the plate. In the observations, the neutral filter was placed over the planetary image, which had to be close to the plate to reduce the size of its penumbra and minimize the knife-edge diffraction caused by the light of the bright primary around the edge of the nichrome filter.

A number of Kodak emulsions were used, including 103aJ, $103 \mathrm{aG}$, and IIIaJ. A fast emulsion was necessary to reduce the exposure times since the planet and satellites were moving rapidly. The 103aJ was preferable since it reduced the bandwidth and thus the atmospheric refraction and dispersion. More importantly, it reduced the extent of the reddish Martian halo in which the satellites were involved. When Kodak stopped manufacturing this emulsion, $103 \mathrm{aG}$ plates were used. While the IIIaJ plates gave sharp images, the contrast was so high that the disk of Mars' image was "lumpy" due to the intensity variations around its limb, such as the polar caps. This adversely affected the disk measurements, so that emulsion was abandoned.

Adjustment of the planet behind the spot filter could be made with the plate cartridge aside. With the shutter closed, the plate cartridge was slid into the focal plane, the shutter opened, and the exposure begun. This arrangement permitted multiple exposures with intermittent adjustments. Usually two or three exposures were taken, separated in RA by a few arc minutes. Generally, for the $103 \mathrm{aJ}$ and $103 \mathrm{aG}$ plates, exposures of $10 \mathrm{~s}, 20 \mathrm{~s}$, and $30 \mathrm{~s}$ were taken on each plate with the 61-inch, and exposures of and $20 \mathrm{~s}, 40 \mathrm{~s}$, and $60 \mathrm{~s}$ with the IIIaJ plates. With the 26-inch, exposures of $20 \mathrm{~s}, 40 \mathrm{~s}$, and $60 \mathrm{~s}$ were taken with the $103 \mathrm{aJ}$ and $103 \mathrm{aG}$ plates. The shortest exposure on each plate yielded a good image of Phobos on a faint planetary halo, but a weak image of Deimos, and the longest exposure provided a stronger image for Deimos. Table 1 summarizes the exposure data for the fourteen oppositions in the series.

\section{Measurement and reduction}

\subsection{Observations with the USNO 61-inch astrometric reflector and plate measurement}

One hundred ninety plates were selected and transmitted to ROB to be digitized (Robert et al. 2011; de Cuyper et al. 2011). Each plate contains two to three exposures shifted in the RA direction. The exposure time of the photographic plates are 10-90 s. The field of view is 37 arcmin on the $x$-axis and 26 arcmin on the $y$-axis. We were able to make measurements for 480 positions of Mars, 357 positions of Phobos, and 371 positions of Deimos. As an example, Fig. 1 shows the center of the digitized (negative) USNO Martian plate No. 02047, which is a typical digitized image. This ten-second exposure of Mars and its moons was taken on 28 May, 1969 in Flagstaff, Arizona. Phobos, the 
Table 1. Raw statistics of the USNO Martian photographic plates that were digitized at ROB.

\begin{tabular}{ccccccccc}
\hline \hline Serie & Instrument & Year & Month & Emulsion & Exp. time $(\mathrm{s})$ & Mars & Phobos & Deimos \\
\hline 01 & $61 \mathrm{i}$ & 1967 & 4 & $103 \mathrm{aG}-103 \mathrm{aJ}$ & $10-40$ & 88 & 67 & 81 \\
02 & $61 \mathrm{i}$ & 1969 & $5-6$ & $103 \mathrm{~J}-\mathrm{IIIJJ}$ & $7-90$ & 283 & 219 & 204 \\
03 & $26 \mathrm{i}$ & 1971 & $8-9$ & $103 \mathrm{aJ}$ & $10-60$ & 95 & 95 & 65 \\
04 & $26 \mathrm{i}$ & 1973 & 10 & $103 \mathrm{aJ}$ & $20-90$ & 94 & 93 & 93 \\
05 & $26 \mathrm{i}$ & 1975 & 12 & $103 \mathrm{aG}-103 \mathrm{aJ}$ & $30-90$ & 141 & 92 & 134 \\
06 & $26 \mathrm{i}$ & 1978 & $1-2$ & $103 \mathrm{aG}$ & $30-60$ & 42 & 33 & 21 \\
07 & $26 \mathrm{i}$ & 1980 & $2-3$ & $103 \mathrm{aG}$ & $30-62$ & 24 & 16 & 9 \\
08 & $61 \mathrm{i}$ & 1982 & 3 & $103 \mathrm{aG}-\mathrm{IIIaJ}$ & $10-50$ & 58 & 35 & 37 \\
09 & $26 \mathrm{i}$ & 1984 & $4-5$ & $103 \mathrm{aG}$ & $40-60$ & 24 & 18 & 20 \\
10 & $26 \mathrm{i}, 61 \mathrm{i}$ & 1986 & 7 & $103 \mathrm{aG}-\mathrm{IIaD}$ & $3-60$ & 66 & 51 & 61 \\
11 & $26 \mathrm{i}$ & 1988 & $9-10$ & $103 \mathrm{aG}$ & $10-90$ & 63 & 60 & 54 \\
12 & $26 \mathrm{i}$ & 1990 & $11-12$ & $103 \mathrm{aG}$ & $30-50$ & 78 & 48 & 57 \\
13 & $26 \mathrm{i}$ & 1995 & 2 & $103 \mathrm{aG}$ & $30-50$ & 21 & 15 & 20 \\
14 & $26 \mathrm{i}$ & 1997 & 3 & $103 \mathrm{aG}$ & $40-45$ & 9 & 5 & 9 \\
\hline \multicolumn{7}{c}{} \\
\hline
\end{tabular}

Notes. The Mars and satellite columns give the numbers of corresponding objects that could be measured on the plates.

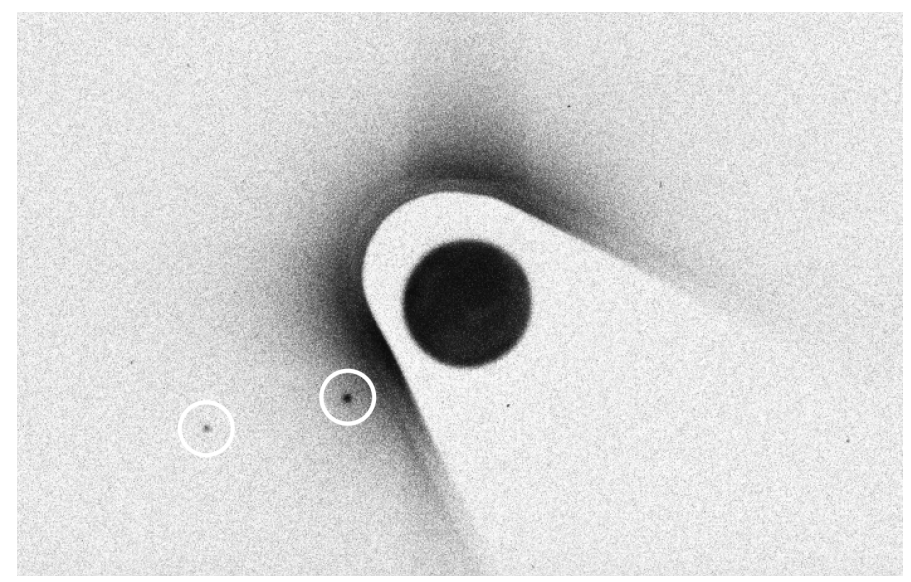

Fig. 1. Center of the digitization (negative) of the USNO 61-inch Martian plate No. 02047. Phobos and Deimos are displayed in the white circle. Phobos is the brighter object here. The dark shape around Mars is the shadow of the small nichrome filter. North is up, east to the left.

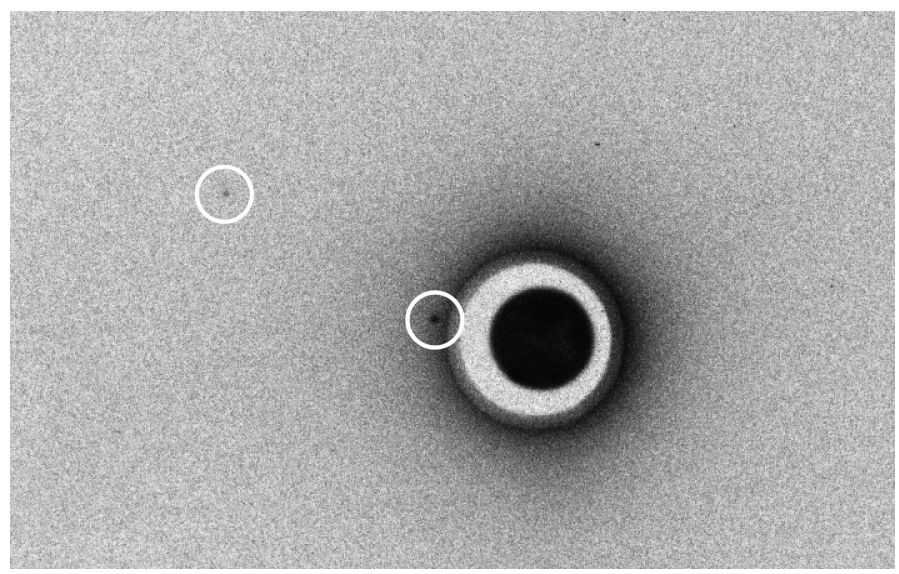

Fig. 2. Center of the digitization (negative) of the USNO 26-inch Martian plate No. 11047. Phobos and Deimos are displayed in the white circle. Phobos is the brighter object here. The dark shape around Mars is the shadow of the small nichrome filter. North is up, east to the left. brighter moon, is southeast of Mars, while Deimos is a little farther to the southeast. The photographic plate was a Kodak 103aJ in combination with a Schott GG14 filter.

Measurement techniques were the same as described in Robert et al. (2014) with small differences because cluster plates were not available for these series. Measured $(x, y)$ plate positions were corrected for instrumental and spherical effects. The observations contain two to nine UCAC4 reference stars (Zacharias et al. 2013), and the reductions were performed here using suitable six constant functional models (Robert 2011; Robert et al. 2011) to provide equatorial (RA, Dec) astrometric positions of the planet and its satellites. Scales $\rho_{x}$ and $\rho_{y}$, orientations $\theta_{x}$ and $\theta_{y}$, and offsets $\Delta_{x}$ and $\Delta_{y}$ were modeled for the determination of the tangential $(X, Y)$ coordinates. All our observations were equatorial (RA, Dec) astrometric positions obtained from tangential $(X, Y)$ coordinates by using the gnomonic inverse projection and determined in an ICRS geocentric reference frame to be easily compared with the most recent ephemerides.

\subsection{Observations with the USNO 26-inch refractor and plate measurement}

Two hundred thirty five plates were selected and transmitted to ROB to be digitized. Each plate contains two to three exposures shifted in the RA direction. The exposure time of the photographic plates are 10-90 s. The field of view is 57 arcmin on the $x$-axis and 43 arcmin on the $y$-axis. We were able to make measurements for 606 positions of Mars, 490 positions of Phobos, and 494 positions of Deimos. As an example, Fig. 2 shows the center of the digitized (negative) USNO Martian plate No. 11047, which is a typical digitized image. This thirty-second exposure of Mars and its moons was taken at the favorable opposition of Mars on 29 September, 1988 in Washington, DC. Phobos, the brighter moon, is east of Mars, while Deimos is farther to the northeast. The photographic plate was a Kodak $103 \mathrm{aG}$ in combination with a Schott GG14 filter.

Measurement and reduction techniques were the same as described in Robert et al. (2014). Measured $(x, y)$ plate positions were corrected for instrumental and spherical effects. The observations contain two to fifteen UCAC4 reference stars, and the reductions were performed using suitable four constant functional models to provide equatorial (RA, Dec) astrometric positions 
Table 2. Extract from the astrometric positions list of Mars, Phobos, and Deimos available in electronic form at the CDS and IMCCE.

\begin{tabular}{cccc}
\hline \hline Object & Date (TDB) & RA $(\mathrm{deg})$ & Dec $(\mathrm{deg})$ \\
\hline Mars & 2442770.734562 & 78.960865 & 26.081444 \\
Phobos & 2442770.734562 & 78.966202 & 26.084275 \\
Deimos & 2442770.734562 & 78.971576 & 26.087503 \\
\hline
\end{tabular}

Notes. The full table is available at the CDS and IMCCE.

of the planet and its satellites. All our observations were equatorial (RA, Dec) astrometric positions obtained from tangential $(X, Y)$ coordinates by using the gnomonic inverse projection and determined in an ICRS geocentric reference frame to be easily compared with the most recent ephemerides.

\section{Positioning results}

We compared the observed positions of Mars, Phobos, and Deimos with their theoretical computed positions given by INPOP13c planetary ephemeris (Fienga et al. 2014) and NOE MarsSatV1_0 satellite ephemerides (Lainey et al. 2007). We then focused on individual observations for which the $(\mathrm{O}-\mathrm{C}) \mathrm{s}$ of the planet and the satellites were independently less than the $3 \sigma$ value of their rms $(\mathrm{O}-\mathrm{C})$ in right ascension and declination. This concerns 777 positions of Mars, 640 positions of Phobos, and 704 positions of Deimos. Positions for Mars were derived indirectly from the satellites, except when they were not measurable then positions for Mars were derived from direct measurements. In the list available in electronic form at the CDS and IMCCE, the corresponding geocentric observed positions refer to the ICRF, and the mean time of observation is given in Barycentric Dynamical Time TDB. Table 2 gives an extract of this list. Starting from the lefthand column, we provide the object name, the mean TDB date of observation in Julian Days, the geocentric observed right ascension, and declination in degrees. The $(\mathrm{O}-\mathrm{C})$ distributions of residuals in equatorial right ascension and declination are provided in Figs. 3 and 4 and in Table 3. They show the difference in (RA, Dec) coordinates for individual planet and satellites, hence the observed positions versus positions calculated from INPOP13c ephemeris.

Offsets for each night of a single opposition set are small, but biases can occur in both the RA and Dec coordinates. We conclude that the exposure timing can be ruled out. The dome clock was calibrated each night to the USNO masterclock, and the camera shutter could be opened and closed on integral seconds with a precision of $0.2-0.3 \mathrm{~s}$. The time of mid-exposure thus has a precision better than $0.5 \mathrm{~s}$. We have already discussed the impact of local systematic errors of the reference star catalogs (Robert 2011; Robert et al. 2011, 2014): the epoch differences between the plates and the central epoch of the reference stars are up to about $25 \mathrm{yr}$, and their expected systematic errors are about 10-50 mas. Observations of each opposition span one to two months, that is to say from $15^{\circ}$ to $30^{\circ}$ along the path of Mars. The sets of reference stars are different, and with about two to fifteen stars per field, the astrometric reduction may be affected by accidental errors of individual reference stars.

Offsets for each opposition set, visible in Fig. 3, are small and can be modeled with a 15.4-yr periodic signal of 16 mas of magnitude. Its periodicity corresponds to the favorable opposition period of Mars. When the planet crosses the ecliptic plane, its relative distance to Earth is either minimum or maximum, and the offset is zero. This indicates that the eccentricity and inclination of the orbit of Mars introduces a height effect. We conclude
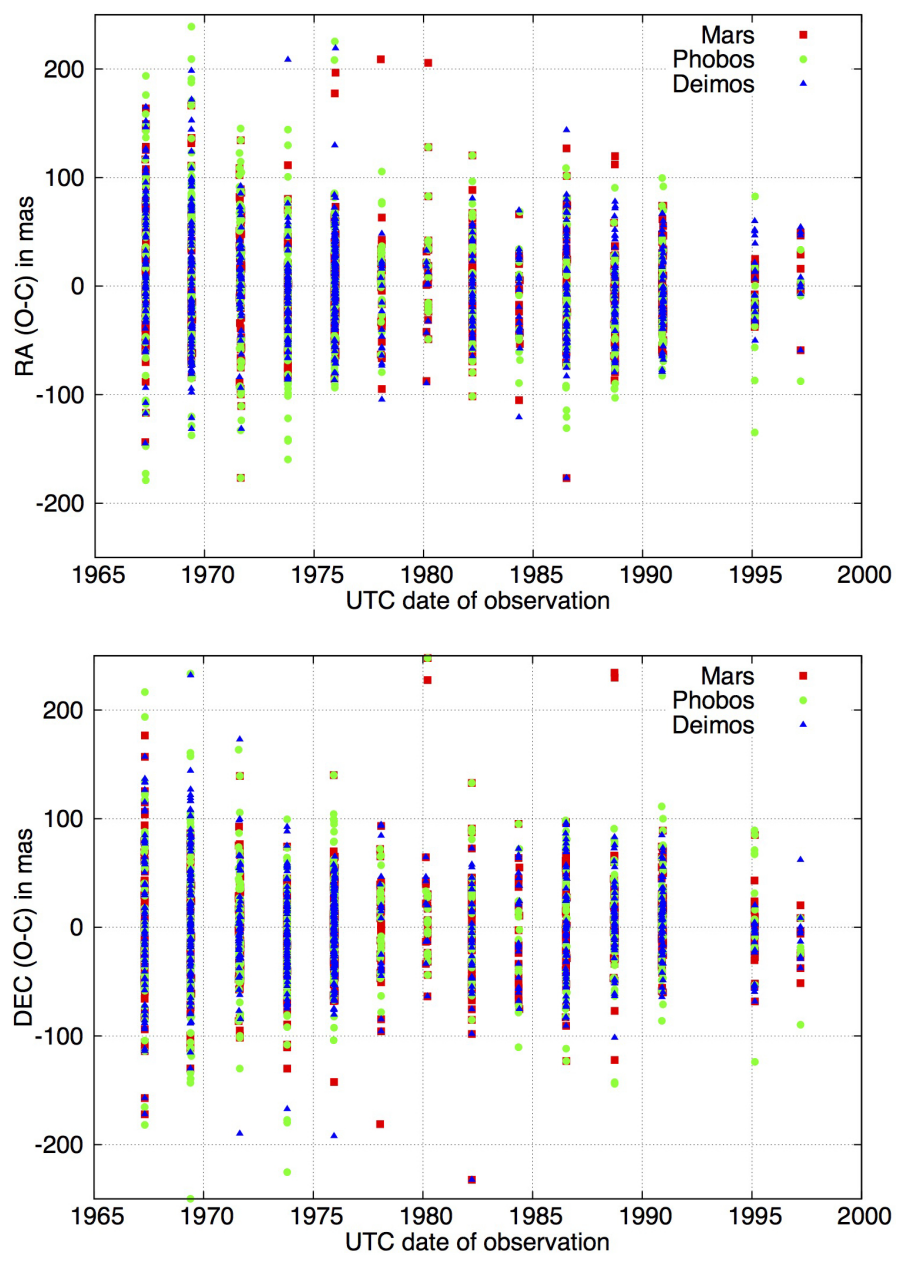

Fig. 3. (RA, Dec) (O-C) according to the NOE MarsSatV1_0 and INPOP $13 \mathrm{c}$ ephemerides. The $x$-axis shows the UTC date of observation and $y$-axis the RA and Dec $(\mathrm{O}-\mathrm{C})$ s. Red squares denote Mars, green circles Phobos, and blue triangles Deimos.

that any mismodeling of instrumental and spherical corrections and, in particular, of the total and differential atmospheric refraction, can be ruled out because the offsets at minimum and maximum distances are zero, so no effect remains. Moreover, the signal is eliminated while dealing with intersatellite residuals. Adjustment and weighting of the planetary ephemeris used could explain this bias, particularly because it is better constrained when the planet crosses the ecliptic plane. We fitted a periodic signal with the (O-C)s according to DE430 ephemeris (Folkner et al. 2014), and the amplitude slightly changed to 15 mas, but observations used for the fit of the numerical integrations leading to INPOP13c and DE430 are quite similar. We also fitted a periodic signal according to older ephemerides for which observations used for the fit and numerical integrations are different. The amplitude of the fitted signal according to DE200 ephemeris (Standish 1982) and VSOP87 ephemeris (Bretagnon \& Francou 1988) changed to 21 mas and 12 mas, respectively.

The average $(\mathrm{O}-\mathrm{C})$ values for the observations made from 1967 to 1997 are very low in RA and Dec coordinates. Furthermore, to estimate the influence of the planetary ephemeris on the results, we computed the difference between observed positions and positions calculated from DE430 ephemeris and from older planetary models and, in particular, from INPOP10e (Fienga et al. 2013), INPOP06 (Fienga et al. 2008), VSOP87, DE421 (Folkner et al. 2009), DE405/DE406 
Table 3. Details of the (RA, Dec) (O-C) in mas according to the NOE MarsSatV1_0 and INPOP13c ephemerides.

\begin{tabular}{ccccccc}
\hline \hline & $\overline{(\mathrm{O}-\mathrm{C})_{\alpha \cos \delta}}$ & $\begin{array}{c}S E M_{\alpha \cos \delta} \\
+/-\end{array}$ & $\sigma_{\alpha \cos \delta}$ & $\overline{(\mathrm{O}-\mathrm{C})_{\delta}}$ & $\begin{array}{c}S E M_{\delta} \\
+/-\end{array}$ & $\sigma_{\delta}$ \\
\hline Mars & 0.5 & 1.6 & 47.3 & -0.2 & 1.7 & 48.3 \\
Phobos & 1.4 & 2.4 & 62.1 & 2.9 & 2.3 & 58.8 \\
Deimos & 2.0 & 1.9 & 50.4 & -0.8 & 1.9 & 50.9 \\
\hline
\end{tabular}

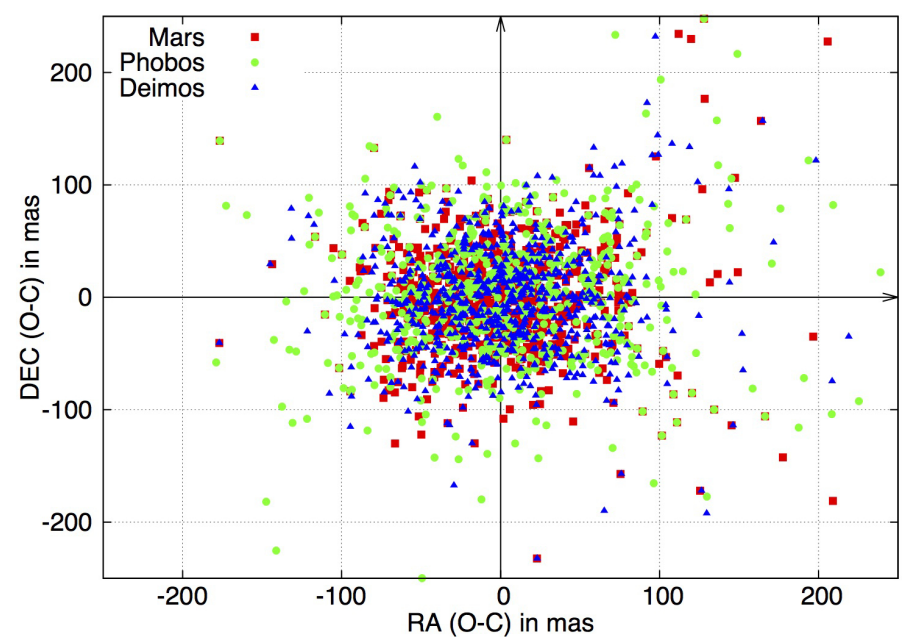

Fig. 4. (RA, Dec) (O-C) according to the NOE MarsSatV1_0 and INPOP13c ephemerides. The $x$-axis shows the RA $(\mathrm{O}-\mathrm{C})$ and $y$-axis the Dec (O-C). Red squares denote Mars, green circles Phobos, and blue triangles Deimos.

(Standish 1998), and DE200 ephemerides. Table 4 shows the difference between the observed positions and positions calculated from the corresponding ephemerides. The mean difference between INPOP13c, INPOP10e, and DE430 for Mars is supposed to be less than 1.2 mas (Fienga et al. 2014), and we may deduce from our results that this value is realistic for both coordinates. We also find a bias up to 15 mas in RA according to VSOP87 ephemeris. Because of the difficulty representing the real motion of Mars with analytical developments, this bias is consistent with the value up to 20 mas given by comparisons between VSOP theory and numerical integrations in Bretagnon (1982) and Bretagnon \& Francou (1988). But it is important to note that these average $(\mathrm{O}-\mathrm{C})$ values are minimized over our thirty-year time span because a large number of observations were taken between successive favorable oppositions of Mars and the periodic evolution alternates between maximum and minimum values. Even if the differences between planetary ephemerides are small, we estimate from the amplitude of the periodic signals we fitted that their mean external error range from 7.6 mas to 13.4 mas. In particular, we estimate that the mean external error of DE430 is 9.5 mas, and 10.2 mas for INPOP13c. We have already discussed the impact of accidental observational errors and mismodeling, and we assume that no observational effect remains at the level of 1 mas (Kaplan et al. 1989; Robert 2011). In other words, such a signal may be introduced by the use of the planetary model itself. In corresponding papers, only differences between planetary models are evaluated. External errors are generally estimated separately by comparison between observations that were not used for the fit and the theories. We there see from old observations that the mean external error of recent planetary ephemerides for Mars could reach 10 mas, that is to say, ten times the internal accuracy officially provided. We already found such a bias with a different system. For example, comparisons with old observations indicate that the external error of most recent planetary ephemerides for Jupiter is up to 22.9 mas, derived from a 12.0-yr periodic signal of 36 mas of magnitude (Robert 2011). Further investigations are planned to confirm deviations with other planetary systems.

We finally compared the observed positions of Phobos and Deimos with their theoretical positions given by the most recent MAR097 JPL ephemerides (Jacobson 2010). Results are quite similar to those given by the NOE MarsSatV1_0 model within 2 mas difference, which is consistent with the expected value. It is interesting to see that these observations are very useful for evaluating the accuracy of the ephemerides of Mars and its satellites over a large time span since they have not been used for the fit of the numerical integrations leading to INPOP and DE models, and to NOE MarsSatV1_0 and MAR097 here.

The key point is that the NOE MarsSatV1_0/INPOP13c rms (O-C) for all observations of Mars is 47.8 mas, 60.4 mas for Phobos, and 50.6 mas for Deimos. These average rms (O-C) correspond to our observation accuracies over thirty years. We already compared the results of the 1971 opposition with previous Earth-based observations in Robert et al. (2014), but we now consider positions over a larger time span. Colas (1992) did intersatellite CCD observations during the 1988 opposition with the one-meter telescope at Pic-du-Midi. He found an overall rms $(\mathrm{O}-\mathrm{C})$ of 60 mas, while our overall intersatellite rms $(\mathrm{O}-\mathrm{C})$ is 39 mas.

We also discussed the factors that contributed to the increase in precision, in particular, the original observation and filtering technique, the accurate and dense star catalog used, and most important, the digitization of the photographic plates. We found an overall $\mathrm{rms}(\mathrm{O}-\mathrm{C})$ of about $53 \mathrm{mas}$, that is to say, $18 \mathrm{~km}$. This could be compared to first spacecraft accuracies: the rms (O-C) of the measurements of Mariner 9 (Duxbury \& Callahan 1989) and Viking 1 and 2 (Duxbury \& Callahan 1988) range between $4.5 \mathrm{~km}$ and $25 \mathrm{~km}$. This could also be compared to more recent spacecraft accuracies: the rms $(\mathrm{O}-\mathrm{C})$ of the measurements of Phobos 2 (Kolyuka et al. 1991) range range between 1 and $16 \mathrm{~km}$, between 4 and $7 \mathrm{~km}$ for MGS (Bills et al. 2005), and between 0.4 and $1.5 \mathrm{~km}$ for Mars Express (Oberst et al. 2006; Pasewaldt et al. 2012). Nevertheless, spacecraft observations used to acquire several images on the same orbit, and the errors on their positions introduce a non-Gaussian noise due to the varying distance to the observed moon and the sometimes small number of observations. The huge benefit of a new treatment of astrophotographic plates is to provide observations that are similar in accuracy to old space data but with a much more Gaussian error and a larger time span.

\section{Orbital adjustment}

\subsection{The NOE numerical model}

The Numerical Orbit and Ephemerides (NOE) software is a general and powerful $N$-body code used to fit initial positions, velocities, and various physical parameters to observation data. 
Table 4. Details of the (RA, Dec) (O-C) in mas according to the NOE MarsSatV1_0 and INPOP10e, INPOP06, VSOP87, DE430, DE421, DE405/DE406, and DE200 ephemerides.

\begin{tabular}{ccccccc}
\hline \hline & $\overline{(\mathrm{O}-\mathrm{C})_{\alpha \cos \delta}}$ & $\begin{array}{c}S E M_{\alpha \cos \delta} \\
+/-\end{array}$ & $\sigma_{\alpha \cos \delta}$ & $\overline{(\mathrm{O}-\mathrm{C})_{\delta}}$ & $\begin{array}{c}S E M_{\delta} \\
+/-\end{array}$ & $\sigma_{\delta}$ \\
\hline INPOP10e & & & & & & \\
Mars & 0.8 & 1.7 & 47.4 & -0.3 & 1.7 & 48.3 \\
Phobos & 1.8 & 2.4 & 62.2 & 2.9 & 2.3 & 58.8 \\
Deimos & 2.3 & 1.9 & 50.5 & -0.8 & 1.9 & 50.9 \\
\hline INPOP06 & & & & & & \\
Mars & -0.3 & 1.6 & 47.3 & 2.8 & 1.7 & 48.1 \\
Phobos & 0.4 & 2.4 & 62.1 & 2.8 & 2.3 & 58.8 \\
Deimos & 0.9 & 1.9 & 50.3 & -0.8 & 1.9 & 50.9 \\
\hline VSOP87 & & & & & & \\
Mars & 16.6 & 2.0 & 54.5 & 5.0 & 1.8 & 49.6 \\
Phobos & 17.2 & 2.6 & 64.8 & 2.3 & 2.3 & 59.7 \\
Deimos & 17.9 & 2.0 & 54.3 & -0.1 & 1.9 & 51.2 \\
\hline DE430 & & & & & & \\
Mars & 0.2 & 1.6 & 47.3 & -0.2 & 1.7 & 48.3 \\
Phobos & 1.2 & 2.4 & 62.2 & 2.9 & 2.3 & 58.8 \\
Deimos & 1.7 & 1.9 & 50.5 & -0.9 & 1.9 & 50.9 \\
\hline DE421 & & & & & & \\
Mars & 0.0 & 1.6 & 47.3 & -0.2 & 1.7 & 48.3 \\
Phobos & 0.9 & 2.4 & 62.2 & 2.9 & 2.3 & 58.8 \\
Deimos & 1.4 & 1.9 & 50.4 & -0.9 & 1.9 & 50.9 \\
\hline DE405/DE406 & & & & & & \\
Mars & -0.3 & 1.7 & 47.4 & -0.2 & 1.7 & 48.3 \\
Phobos & 0.8 & 2.4 & 62.3 & 3.1 & 2.3 & 58.0 \\
Deimos & 1.2 & 1.9 & 50.5 & -1.0 & 1.9 & 50.9 \\
\hline DE200 & & & & & & \\
Mars & 1.8 & 1.6 & 47.3 & 0.2 & 1.8 & 49.3 \\
Phobos & 1.3 & 2.4 & 61.6 & 1.8 & 2.3 & 59.3 \\
Deimos & 2.9 & 1.9 & 50.3 & -0.9 & 1.9 & 51.3 \\
\hline
\end{tabular}

The modeling used in this work is similar to what is used in Lainey et al. (2007) with small differences: we here consider the MRO110c Martian gravity field that is an update to the gravity fields obtained in Konopliv et al. (2011), the perturbations of the Sun, Jupiter, Saturn, the Earth, and the Moon using DE430 ephemerides, the MRO110c mass of the Martian moons, and the Martian precession and rotation obtained in Kuchynka et al. (2014). The dynamical system was integrated in a planetocentric frame with inertial axes. A constant step size of $\Delta t=0.020$ day was used during the integrations that were performed over one century before and after the initial Julian epoch of 2451545.0 (01/01/2000 at 12h00 TDB), to keep ten meters of precision for the spacecraft era.

\subsection{Fits to the observations}

To assess the importance of our large data set, we decided to test our positional results by means of two different fits. The first numerical fit, which we call NOE_1, only considered space data. This data set consists in Mariner 9, Viking 1 and 2, Phobos 2, Mars Express, and Mars Reconnaissance Orbiter (Jacobson, priv. comm.) observations. The 643 positions of Phobos and 733 positions of Deimos were used, spanning 1971 to 2011. The second numerical fit, which we call NOE_2, considered the USNO photographic plate data and the space data only made after 2000. Mars Express and Mars Reconnaissance Orbiter observations were thus introduced. The 1035 positions of Phobos and 1319 positions of Deimos were used, respectively spanning from 1967 to 1997 and 2004 to 2011. Because spacecraft data are more accurate and even if fewer observations were taken into account in the first data set, differences between the two fits will allow assessing the accuracy of old astrophotographic plate data compared to space data. The initial epoch was also chosen in consequence.

\subsection{Adjustment results}

Figure 5 shows differences in distance between the NOE_1 and NOE_ 2 models for Phobos and Deimos. The average difference for Phobos is about of $2.8 \mathrm{~km}$ over one century but the resulting differences can reach $13.3 \mathrm{~km}$ after $50 \mathrm{yr}$ of propagation backward to the initial epoch. This divergence may be explained by a small difference in the mean motions, transcribing a tiny difference in the satellite velocity between the two models. But the key point is that the average difference in distances $30 \mathrm{yr}$ back to the initial epoch is about of $5 \mathrm{~km}$. This epoch corresponds to that of the Mariner 9 mission and Viking program, and it shows that the two fits are as accurate.

The average difference for Deimos is about of $0.1 \mathrm{~km}$ over one century and the resulting differences are less than $1 \mathrm{~km}$. This result is consistent with what was expected, in particular because its dynamics is simpler thanks to its larger distance from the primary.

The fastest divergence of Phobos and the better constrained solution for Deimos are confirmed with Figs. 6 and 7. Figure 6 shows differences in semi-major axis between the NOE_1 and NOE_2 models for Phobos and Deimos. Figure 7 shows corresponding differences in longitude. The average difference in semi-major axis over one century is about of $0.005 \mathrm{~m}$ for Phobos, and $0.001 \mathrm{~m}$ for Deimos. The average difference in longitude over one century is about of $2.8 \mathrm{~km}$ for Phobos and $0.0 \mathrm{~km}$ for Deimos. Moreover, the evolution provided in Fig. 7 is a typical 
V. Robert et al.: USNO astrometric observations of Phobos and Deimos
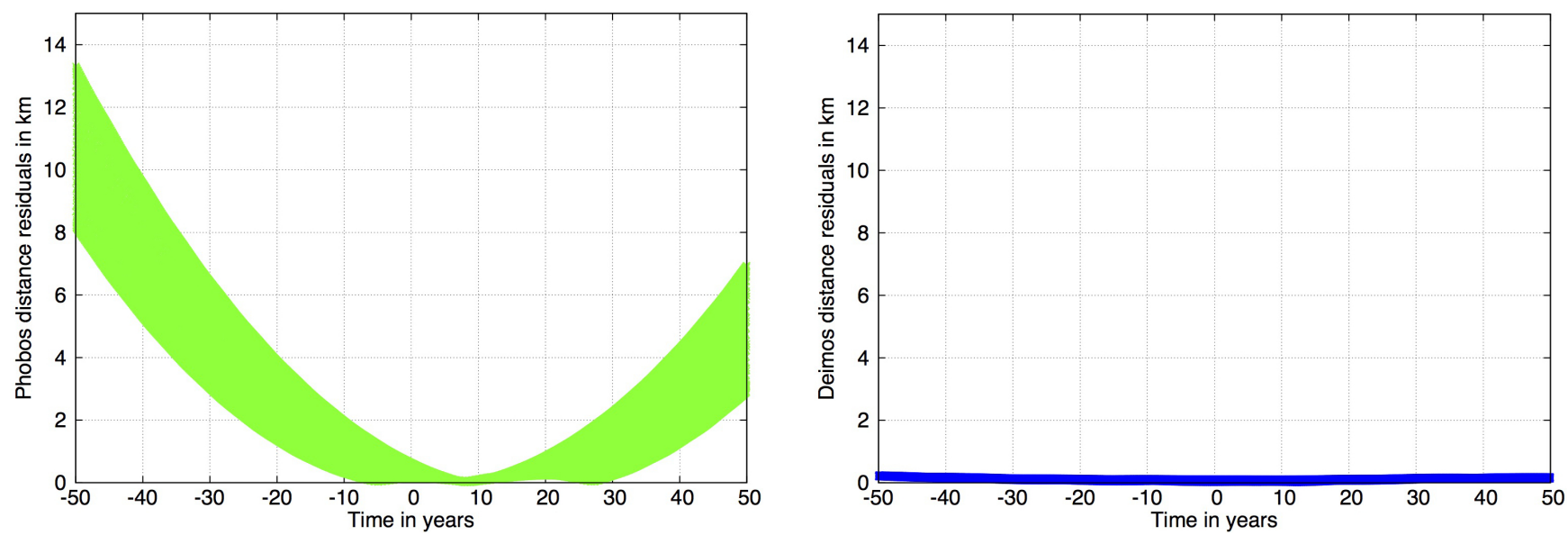

Fig. 5. Differences in distance between the NOE_1 and NOE_2 models for Phobos (left panel) and Deimos (right panel). The $x$-axes are relative in years to the Julian epoch of 2451545.0 , and the $y$-axes are distance residuals in km.
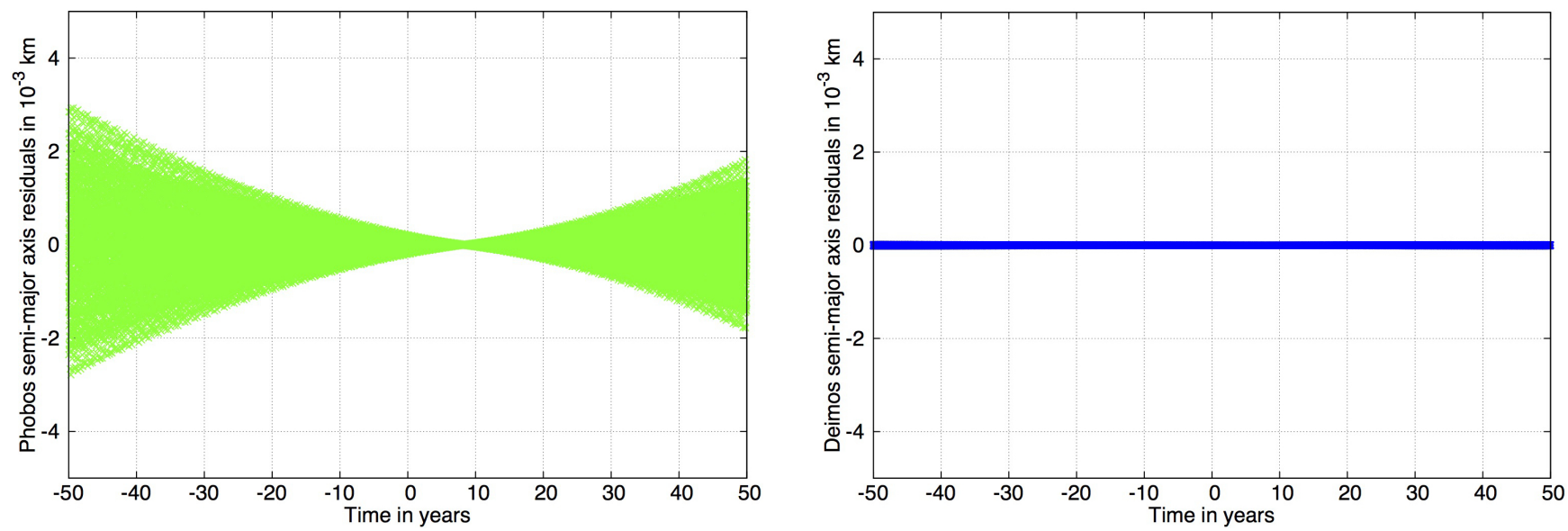

Fig. 6. Differences in semi-major axis between the NOE_1 and NOE_2 models for Phobos (left panel) and Deimos (right panel). The $x$-axes are relative in years to the Julian epoch of 2451545.0 , and the $y$-axes are semi-major axis residuals in $10^{-3} \mathrm{~km}$.
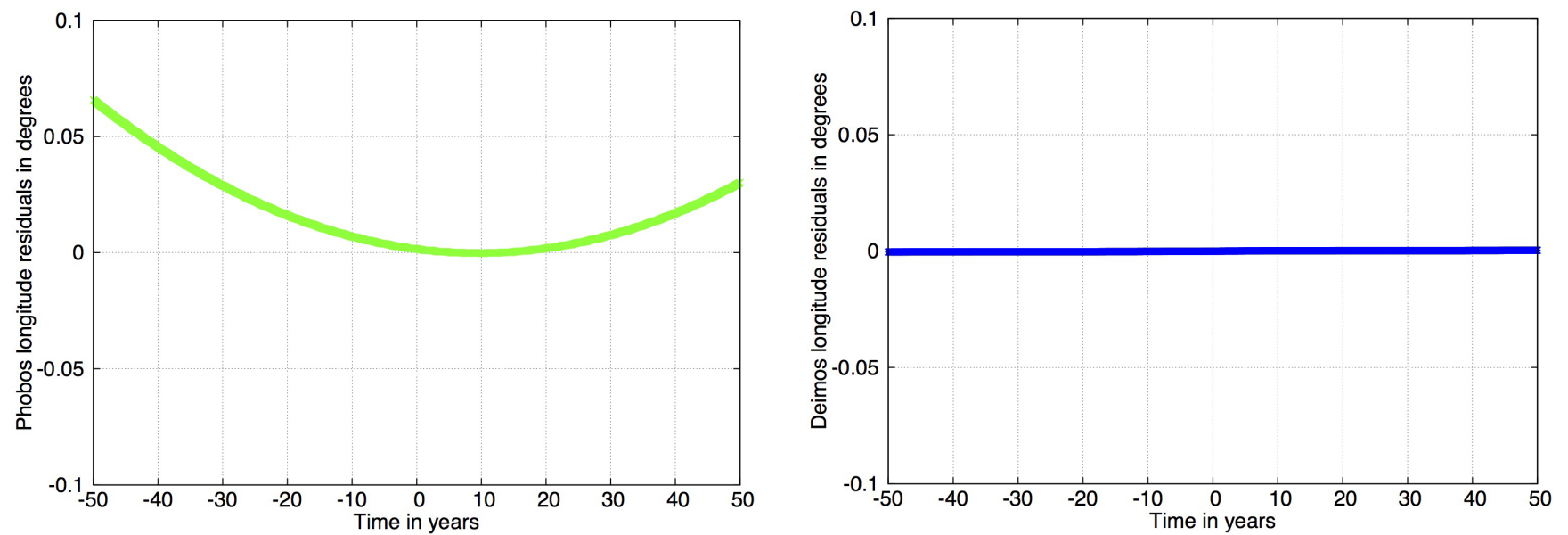

Fig. 7. Differences in longitude between the NOE_1 and NOE_2 models for Phobos (left panel) and Deimos (right panel). The $x$-axes are relative in years to the Julian epoch of 2451545.0 , and the $y$-axes are longitude residuals in degrees.

tidal effect. This corroborates the difference in dynamics due to the observations used for the fits of the NOE_1 and NOE_2 models. And first comparisons of the differences in distances, semimajor axes, and longitudes between the two models show consistent and close results.

We finally come to one of the most interesting goals for studying the orbital evolution of natural satellites: the investigation of the tidal dissipation factor $Q$ of the planet. In the case of the Martian system in particular, the investigation of this factor has been quite important for improving the orbital models. It also allowed to explain the secular acceleration in the longitude of Phobos that was first discovered by Sharpless (1945). Moreover, the Martian tidal dissipation needs a long time span to be estimated accurately. We benefit from such a substantial data 
set, and we were able to fit a $Q$ parameter for both adjustments. Assuming $k_{2}=0.176$ for the Martian Love number (Konopliv et al. 2011; Kuchynka et al. 2014), we found $Q=99.5 \pm 0.9$ for the NOE_1 model and for the NOE_2 model, $Q=101.1 \pm 1.2$.

If we only consider the space data made after 2000, we find $Q=110.8 \pm 9.0$. We can see that the old photographic plate data contribute in the same way as the old spacecraft data by decreasing the error bars significantly with comparable accuracy.

\section{Conclusion}

We analyzed a full series of astrophotographic plates of the Martian satellites taken at USNO from 1967 to 1997 . Thanks to the submicrometric digitization, it is now possible to identify all the available stars (depending on the catalog used) and, more important, those that are not detected by eye. In addition and thanks to the new astrometric catalogs, we were able to provide astrometric (RA, Dec) positions with never achieved accuracy for the planet and its moons from the ground. The rms residuals in RA and Dec of one position are less than 62 mas, or about $20 \mathrm{~km}$ at the opposition of Mars. This provides a positioning accuracy that is very close to that of old spacecraft but a more numerous spread on a longer interval of time with much more Gaussian error. We obtained consistent and close results while studying the dynamics of the satellites over one century. We were also able to estimate the precision on the Martian tidal dissipation factor $Q$, and our results show that astrometric data derived from photographic plates can compete with those of old spacecraft.

It is also important to remember that many useful observations are available in most of the observatories and national archives. We have started to establish contacts to improve the dynamics of the planetary systems with old data in the framework of the FP7 ESPaCE program and ahead of the upcoming NAROO project ${ }^{1}$ of the IMCCE. We also look forward to the arrival of the Gaia reference star catalog. Star catalogs provide accurate positions of the references, but their less accurate proper motions do not permit to analyze photographic plates older than fifty years. Because Gaia proper motions will be welldetermined, reductions of old observations will yield increased accuracy by eliminating any errors caused by the reference star positions. Right ascension and declination of the objects will be perfectly calculated. We estimate that we will be able to decrease the USNO rms residuals in RA and Dec of one position by 15-20 mas, or about $6 \mathrm{~km}$ at the opposition of Mars.

Acknowledgements. The research leading to these results has received funding from the European Community's Seventh Framework Program (FP7/2007-2013) under grant agreement No. 263466 for the FP7-ESPaCE program.

\section{References}

Arlot, J.-E., Desmars, J., Lainey, V., \& Robert, V. 2012, Planet. Space Sci., 73, 66

Bills, B. G., Neumann, G. A., Smith, D. E., \& Zuber, M. T. 2005, J. Geophys. Res. (Planets), 110, 7004

Bretagnon, P. 1982, A\&A, 114, 278

Bretagnon, P., \& Francou, G. 1988, A\&A, 202, 309

Burns, J. A. 1972, Rev. Geophys. Space Phys., 10, 463

Colas, F. 1992, A\&AS, 96, 485

de Cuyper, J.-P., de Decker, G., Winter, L., \& Zacharias, N. 2011, in Astronomical Data Analysis Software and Systems XX, eds. I. N. Evans, A. Accomazzi, D. J. Mink, \& A. H. Rots, ASP Conf. Ser., 442, 301

Desmars, J., Arlot, S., Arlot, J.-E., Lainey, V., \& Vienne, A. 2009, A\&A, 499, 321

Duxbury, T. C., \& Callahan, J. D. 1988, A\&A, 201, 169

Duxbury, T. C., \& Callahan, J. D. 1989, A\&A, 216, 284

Fienga, A., Manche, H., Laskar, J., \& Gastineau, M. 2008, A\&A, 477, 315

Fienga, A., Manche, H., Laskar, J., Gastineau, M., \& Verma, A. 2013, ArXiv e-prints [arXiv: 1301.1510]

Fienga, A., Manche, H., Laskar, J., Gastineau, M., \& Verna, A. 2014, IMCCE, Observatoire de Paris

Folkner, W. M., Williams, J. G., \& Boggs, D. H. 2009, Interplanetary Network Progress Report, 178, C1

Folkner, W. M., Williams, J. G., Boggs, D. H., Park, R. S., \& Kuchynka, P. 2014, Interplanetary Network Progress Report, 196, C1

Hall, A. 1878, Observations and orbits of the satellites of Mars (Washington DC: GPO)

Jacobson, R. A. 2010, AJ, 139, 668

Kaplan, G. H., Hughes, J. A., Seidelmann, P. K., Smith, C. A., \& Yallop, B. D. 1989, AJ, 97, 1197

Kolyuka, Y., Tikhonov, V., Ivanov, N., et al. 1991, A\&A, 244, 236

Konopliv, A. S., Asmar, S. W., Folkner, W. M., et al. 2011, Icarus, 211, 401

Kuchynka, P., Folkner, W. M., Konopliv, A. S., et al. 2014, Icarus, 229, 340

Lainey, V., Dehant, V., \& Pätzold, M. 2007, A\&A, 465, 1075

Morley, T. A. 1989, A\&AS, 77, 209

Oberst, J., Matz, K. D., Roatsch, T., et al. 2006, A\&A, 447, 1145

Pascu, D. 1977, in Planetary Satellites, ed. J. A. Burns, IAU Colloq., 28, 63

Pascu, D. 1978, Vistas Astron., 22, 141

Pascu, D. 1979, in Natural and Artificial Satellite Motion, eds. P. E. Nacozy, \& S. Ferraz-Mello, 17

Pascu, D. 2012, in Proc. NAROO-Gaia Workshop: A new reduction of old observations in the Gaia era

Pasewaldt, A., Oberst, J., Willner, K., et al. 2012, A\&A, 545, A144

Robert, V. 2011, Ph.D. Thesis, Observatoire de Paris

Robert, V., de Cuyper, J.-P., Arlot, J.-E., et al. 2011, MNRAS, 415, 701

Robert, V., Lainey, V., Pascu, D., et al. 2014, A\&A, 572, A104

Robin, A. C., Luri, X., Reylé, C., et al. 2012, A\&A, 543, A100

Sharpless, B. P. 1945, AJ, 51, 185

Standish, Jr., E. M. 1982, A\&A, 114, 297

Standish, Jr., E. M. 1998, JPL, Interoffice Memorandum

Wilkins, G. A. 1966, in The Theory of Orbits in the Solar System and in Stellar Systems, ed. G. I. Kontopoulos, IAU Symp., 25, 271

Wilkins, G. A. 1967, in Mantles of the Earth and Terrestrial Planets, ed. S. K. Runcorn, 77

Wilkins, G. A. 1970, The problems of the satellites of Mars, 29

Zacharias, N., Finch, C. T., Girard, T. M., et al. 2013, AJ, 145, 44

\footnotetext{
1 See the NAROO webpage at http://www.imcce.fr/hosted_ sites/naroo/
}

A36, page 8 of 8 